\title{
Perfil da Prescrição de Fitoterápicos em uma Farmácia de Manipulação de Vitória da Conquista-BA entre 2014 a 2018
}

\author{
Marcelo Batista Moraes ${ }^{1}$; Matheus Santos Marques² ; Erika Chuluk Silva Soares ${ }^{3}$; \\ Rodrigo Santos Damascena
}

\begin{abstract}
Resumo: Os fitoterápicos são medicamentos obtidos através de uma planta medicinal, ou dos seus derivados, com exceção das substâncias isoladas, tendo o objetivo profilático, curativa ou paliativa. O presente trabalho teve como foco verificar o perfil da prescrição de fitoterápico em uma farmácia de manipulação de Vitória da Conquista-Ba entre 2014 a 2018, conhecer o público que mais utiliza dessa terapia, as especialidades dos prescritores desses medicamentos e avaliar a evolução das vendas nos últimos cinco anos. A pesquisa foi do tipo documental/ digital, retrospectiva e descritiva, utilizando o programa Fórmula Certa como fonte de pesquisa, tendo como base de análise as prescrições de medicamentos fitoterápicos referentes ao período de Julho de 2014 a julho de 2018. Os resultados foram verificados utilizando análise percentual e expressos em gráficos e tabelas. Após análise viu-se que todas as prescrições (17.078) apresentavam o nome do usuário; e destas 13.320,84 foram prescritas para mulheres $(78 \%)$ e $3.757,16(22 \%)$ para homens. A área profissional que mais apresentaram prescritores foram nutricionistas com (202), seguido por clínico geral (142), ginecologista (36), cardiologista (19), dermatologista (18), pediatra (16) gastroenterologista (10), endocrinologista (09). Os fitoterápicos com maior frequência foram: Aesculus hippocastanum, Vitis vinífera,Tribullus terrestres, Carapa guaianesis Aubl, Equisetum arvense L. A forma farmacêutica com maior volume de prescrição foi de cápsulas que pertence ao laboratório de sólidos. Verificou- se nos últimos cinco anos uma redução nas vendas dos fitoterápicos.
\end{abstract}

Palavras-chave: Fitoterápicos, farmácia magistral, prescrições.

\section{Profile of the Phytotherapics Prescription in a Pharmacy of Manipulation of Vitória da Conquista in Bahia State between 2014 to 2018}

Abstract: Herbal medicines are medicines obtained through a medicinal plant, its derivatives, with the exception of the isolated substances, with the aim of prophylactic, curative or palliative. The present study aimed to verify the profile of phytotherapeutic prescription in a pharmacy of manipulation of Vitória da Conquista-Ba between 2014 and 2018, to know the public that uses this therapy the most, the specialties of the prescribers of these medicines and to evaluate the sales evolution in the last five years. The research was documentary digital, retrospective and descriptive, using the program Formula Certa research source, based on the prescriptions of herbal medicines referring to the period from July 2014 to July 2018. The results were verified using analysis and expressed in graphs and tables. After analysis it was seen that all the prescriptions $(17,078)$ had the name of the user; and of these 13,320.84 were prescribed for women (78\%) and 3,757.16 (22\%) for men. The professional area that most presented prescribers was nutritionists (202), followed by general practitioner (142), gynecologist (36), cardiologist (19), dermatologist (18), pediatrician (16) gastroenterologist (10), endocrinologist . The most frequent phytotherapics were: Aesculus hippocastanum, Vitis vinifera, Tribullus terrestris, Carapa guaianesis Aubl, Equisetum arvense $L$. The pharmaceutical form with the highest volume of prescriptions was capsules belonging to the solids laboratory. There has been a reduction in sales of herbal medicines in the last five years.

Keywords: Phytotherapics, compounding pharmacy, prescriptions.

\footnotetext{
${ }^{1}$ Graduado em Farmácia pela Faculdade Independente do Nordeste.Contato: marcelomoraes.quimico@hotmail.com;

${ }^{2}$ Graduado em Farmácia pela Universidade Federal da Bahia - UFBA. Especialista em Saúde Pública pela UNINTER.

Contato: matheusmarques@fainor.com.br;

${ }^{3}$ Graduada em Farmácia pela Universidade Tiradentes - UNIT. Especialista em Farmácia de Manipulação pela Racine.

Contato: erika_chuluk@hotmail.com;

${ }^{4}$ Graduação em Farmácia pela UEFS Mestrado em Saúde Pública pela Fiocruz. Contato: rodrigodamascena@fainor.com.br
} 


\section{Introdução}

Pode compreender o medicamento fitoterápico como aquele que é obtido através de uma planta medicinal, ou através dos seus derivados, com exceção das substâncias isoladas, tendo o objetivo profilático, curativa ou paliativa (BRASIL, 2011).

O Brasil é o país que possui o maior percentual de biodiversidade, entre 15 a $20 \%$ do total mundial de toda a flora. Sendo assim, o país possivelmente é o detentor de grandes benéficos para o desenvolvimento da fitoterapia, isso porque possui grande biodiversidade, conhecimento popular e tradicional da utilização das plantas, tecnologia para fazer uma cientifica avaliação desse conhecimento (MAPA, 2006).

No tocante das plantas, principalmente as medicinais, devemos ainda salientar que o conhecimento adquirido sobre estas espécies, a sua utilização, indicações e manejo se apresentam como uma herança dos antepassados, que tradicionalmente, têm transmitido os seus conhecimentos de geração para geração, desde os tempos mais remotos até hoje (LIMA, 2006).

Entre os variados métodos da medicina natural, a fitoterapia é, indubitavelmente, o mais antigo, o mais pesquisado e o que conta com o melhor resultado. Através do desenvolvimento da ciência e o aperfeiçoamento dos estudos sobre o tema, os estudiosos procuraram obter a resposta para a seguinte pergunta: por que as plantas podem curar? Através desse questionamento, as plantas começaram a serem averiguadas no contexto da composição química e não somente como um ser místico. Chegando assim a descoberta de que cada planta possui princípios ativos que são capazes de gerar efeitos (benéficos ou colaterais) no momento em que são introduzidos em outros seres vivos (BRUNO; NALDI, 1998).

Segundo a concepção de Almeida (1993), as plantas medicinais acabaram sendo os primeiros recursos terapêuticos que foram usados com o intuito de cuidar da saúde dos seres humanos e também de sua família, sendo, desta forma, um conhecimento milenar que está agregado na evolução humana, isso porque antes mesmo do surgimento da escrita, os indivíduos já utilizavam as plantas, em momentos como remédio, em outros como alimento.

Sendo assim, os chineses, egípcios, hindus e gregos são vistos como as primeiras civilizações que catalogaram as ervas medicinais, apontando a sua classificação se fundamentando em sua forma, cor, sabor e aroma, agregando nisso as ligações com os astros e, especialmente, por conta dos seus atributos mágicos. (LIMA, 2006).

Analisando a literatura, podemos encontrar diversas citações de povos, onde estes realizaram alguma espécie de utilização de ervas, tanto benéficas como também maléficas. Os 
druidas, sacerdotes celtas, utilizavam as suas poções mágicas, ervas venenosas, fazendo ainda a idealização de um horóscopo se fundamentando na energia das árvores, se embasando nas diferentes épocas do ano (BRUNO; NALDI, 1998).

No contexto do Brasil, o uso das plantas não somente como um alimento, entretanto ainda como uma fonte terapêutica começou desde que os primeiros habitantes que aqui chegaram, por volta de 12 mil anos atrás, originando os paleonídeos amazônicos, onde estes se derivaram das mais importantes tribos indígenas do país. Pouco, entretanto, é conhecido sobre este momento, somente as pinturas rupestres (SILVA, 2004).

No final do século XVIII, surgiu no Brasil um dos mais relevantes contribuidores para as pesquisas de fitoterapia brasileira, Bernardinho Antonio Gomes, médico português que chegou à cidade do Rio de Janeiro, como médico pessoal da princesa Leopoldina. Segundo os estudos de Gomes (1972), Bernardinho ficou muito interessado em pesquisar a quantidade de fitoterápicos extraídos das matas brasileiras e passou a ficar focado em catalogar botanicamente de tais plantas, assim como estudar os seus princípios ativos.

Em meados de 1800, a medicina convencional, que usava meramente as plantas medicinais, se fundamentando inquestionavelmente em todos os livros-texto clássicos de farmacologia. Após então a década de 40 do século passado, o uso de drogas vegetais se direcionou para o plano de terapêutica alternativa, em um contexto de segunda categoria, resultante das evoluções alcançadas através da síntese química de fármacos e também do grande desenvolvimento das indústrias farmacêuticas, somado ao capitalismo (SCHULZ et. al., 2002).

Além da utilização das plantas como matéria-prima, para as preparações (dentre essas os chás, óleos e extratos variados) da Medicina Tradicional ou popular em inúmeros países, as plantas medicinais têm seu aproveitamento obtido, assim também, através do desenvolvimento de fitoterápicos, assim como a partir da extração, isolamento e purificação de compostos vegetais, com o intuito de elaborar fitofármacos (DI STASI, 1996; YUNES et al., 2001).

Sendo assim, a fitoterapia, mesmo apresentando a sua ação se fundamentando nos conceitos farmacológicos da alopatia, sendo assim associada à teoria da causalidade da doença e seu combate, conta com peculiaridades que nos levam para uma noção mais integral do corpo e de seus cuidados (BRASIL, 2006).

Desta forma, a fitoterapia é muito difundida nos dias de hoje em todo o mundo como um procedimento natural preventivo, conservador, regenerador e curativo. $\mathrm{O}$ reconhecimento do seu real valor como um recurso clínico, farmacêutico e econômico levaram diversos países a utilizar a prática deste como política pública de saúde. 
No tocante do Brasil, desde a institucionalização do Sistema Único de Saúde (SUS) através da constituição de 1988, determinando então a saúde como um direito do cidadão e responsabilidade do Estado, começou assim uma nova etapa no contexto político e institucional de saúde em todo o Brasil, resultando assim em várias modificações que tornaram possível a implementação de práticas inovadoras na gestão em saúde, entre estas, podemos destacar as de inclusão de algumas das medicinas não-convencionais e práticas complementares para os serviços assistenciais médicos que são prestados para a população (PINHEIRO; LUZ, 2003).

Este trabalho visou traçar o Perfil da prescrição de fitoterápicos em uma farmácia de manipulação de Vitória da Conquista-BA entre 2014 a 2018. Verificando quantas prescrições foram realizadas neste período, assim como a proporção entre médicos e nutricionistas. Quais os principais fitoterápicos mais vendidos na empresa. Sua forma farmacêutica e a evolução no período de cinco anos.

\section{Metodologia}

Esse trabalho foi desenvolvido em uma farmácia de manipulação da cidade de Vitória da Conquista-BA com o foco na avaliação do Perfil da prescrição de fitoterápicos no período limitado em 2014 a 2018.

A pesquisa foi do tipo documental em meio eletrônico utilizando o programa Fórmula Certa que consiste no banco de dados que a farmácia pode contar com o suporte técnico e o suporte farmacoterapêutico, cadastros de clientes, orçamentos e todos os registros da rotina da empresa no aspecto retrospectivo e descritivo, tendo como instrumento os registros que o sistema possui e como objeto de estudo prescrições de medicamentos fitoterápicos referentes ao período de Julho 2014 a Julho de 2018. Foram avaliados os medicamentos fitoterápicos mais prescritos e dispensados na farmácia de manipulação. Assim como sua forma farmacêutica e as proporções entre médicos e nutricionistas nas prescrições dos mesmos. Como a pesquisa não envolveu seres humanos, mas documentos armazenados no programa Fórmula Certa, no caso prescrições de 2014 a 2018, não foi necessário obter o Termo de Consentimento Livre e Esclarecido. O nome dos usuários e dos prescritores não foram revelados.

A avaliação foi das formas farmacêuticas mais dispensadas nesse período, especialidade médica presente, quais fitoterápicos mais prescritos nas receitas. Os dados foram avaliados utilizando gráficos, percentuais e tabelas. Para obter os dados junto a Farmácia de 
manipulação foi necessário que o Programa Fórmula Certa estivesse com seu Banco de dados atualizados.

Para a elevação da pesquisa foram utilizados livros sobre fitoterápicos, artigos científicos nas bases de dados Scielo, Pubmed, Google Acadêmico. Utilizando como palavras chaves: Fitoterápicos, plantas medicinais, farmácia de manipulação, sem restringir o idioma, limitando a data da pesquisa em 2011 a 2018.

\section{Resultados e Discussão}

\section{Identificação do Usuário}

Conforme análise dos dados armazenados no Sistema Fórmula Certa, todos os cadastros continham o nome do usuário. Esse resultado está de acordo com RDC n ${ }^{\circ}$ 67, de 8 de Outubro de 2007 que determina a obrigatoriedade do nome completo do usuário. Esta identificação do paciente não condiz com uma pesquisa em duas farmácias na cidade de Araraquara e Ouro Preto onde apresentaram um valor de 0,22 e 3,5\% de não identificação do paciente respectivamente (MASTROIANNI, 2009; SEBASTIÃO, 2002). Esse número pode ser justificado pelo fato do profissional farmacêutico não ter um controle e avaliação rígida sobre esse ponto.

\section{Gênero do Usuário}

Entre os usuários citados nas prescrições (17.078) que utilizaram este tipo de tratamento, $3.757,16(22 \%)$ correspondem ao sexo masculino e $13.320,84(78 \%)$ ao sexo feminino conforme gráfico 1. A proporção do número de mulheres confirma uma maior aceitabilidade dessa terapia, bem como ao fato das mulheres procurarem mais os serviços de saúde do que os homens (PINHEIRO, et al., 2002). De acordo com análise realizada na cidade de Belo Horizonte a similaridade da pesquisa, mostrou que $74,8 \%$ para mulheres e $25,2 \%$ para homens, reafirmando uma maior aceitabilidade dessa terapia por mulheres (RIBEIRO, LEITE, DANTAS-BARROS, 2005). 


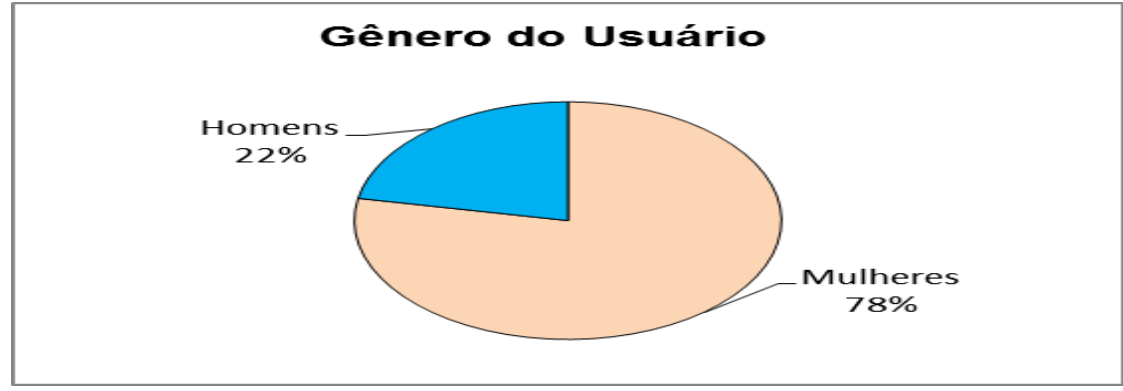

Gráfico 1. Percentual de Gênero que adquiriram medicamentos fitoterápicos na farmácia de manipulação no período de 2014 a 2018.

\section{Idade do Consumidor}

Não foi possível obter essa informação, pois o campo de preenchimento desse dado não estava sendo executado pela equipe da recepção no ato da compra do produto. Essa informação pode não ter sido preenchida pelo fato de não ser um item obrigatório na prescrição e que seja exigido para a dispensação. Segundo a lei 5991/73 os itens indispensáveis para aviar uma prescrição são: necessidade de estar escriturada a tinta, em vernáculo, por extenso e de modo legível, ou digitalizada, conter o nome e o endereço residencial do usuário e, expressamente, o modo de usar o medicamento; conter a data e a assinatura do profissional, endereço do consultório ou da residência, e o número de inscrição no respectivo Conselho profissional (BRASIL, 1973; BRASIL, 2001).

\section{Perfil do Prescritor}

Durante a pesquisa pode-se observar que foi notificado à especialidade do profissional de saúde e seu código de inscrição junto ao conselho profissional para evitar uma possível duplicidade na contagem dos dados.

Após a pesquisa e análises dos dados foram contabilizados 453 profissionais prescritores dispostos na tabela 1. Dentre as especialidades citadas, foi detectado um número considerável de prescrições por Médico não Identificado, onde se classifica como aquele consumidor que chega ao balcão da farmácia com a receita ilegível do carimbo ou assinatura do prescritor ou sendo indicado pelo Farmacêutico do estabelecimento.

A área profissional que mais apresentou prescritores foi nutricionistas com (202) prescritores, seguido por clínico geral (142), ginecologista (36), cardiologista (19), 
dermatologista (18), pediatra (16) gastroenterologista (10), endocrinologista (09), médico não identificado (01).

Ao realizar a soma das porcentagens do total de prescrições para cada classe profissional, os médicos e médico não identificados correspondem a $65,05 \%$ das prescrições e $34,95 \%$ correspondem às prescrições realizadas por nutricionistas. De acordo com a pesquisa realizada no Rio Grande do Sul, os

profissionais com maiores prescrições foram clínico geral $(42,26 \%)$, cardiologista $(16,49 \%)$, psiquiatra $(15,46 \%)$, ginecologista $(5,15 \%)$ e neurologista $(5,15 \%)$ (SILVA, BANDEIRA, OLIVEIRA, 2012).

Esses dados podem está relacionados de acordo com o perfil socioeconômico da população, acesso ao profissional nutricionista e políticas públicas.

Tabela 1: Número de profissionais prescritores de fitoterápicos por especialidade no período de 01/07/2014 a 01/07/2018.

\begin{tabular}{|l|c|}
\hline \multicolumn{1}{|c|}{ Especialidades } & Número de profissionais prescritores \\
\hline Nutricionista & 202 \\
\hline Clínico Geral & 142 \\
\hline Ginecologista & 36 \\
\hline Cardiologista & 19 \\
\hline Dermatologista & 18 \\
\hline Pediatra & 16 \\
\hline Gastroenterologista & 10 \\
\hline Endocrinologista & 09 \\
\hline Médico não Identificado & 01 \\
\hline Total de Prescritores & $\mathbf{4 5 3}$ \\
\hline
\end{tabular}

Fonte: Pesquisa dos autores, 2018.

\section{Os Cinco Fitoterápicos mais prescritos por período anual}

De acordo com a análise dos registros do programa Fórmula Certa foi verificado os cinco fitoterápicos mais prescritos no período de cinco anos, onde encontramos praticamente a repetição dos mesmos em todos os períodos, tendo como diferença apenas sua posição em relação aos anos anteriores. 
Estudos feitos na cidade do Mato Grosso em uma farmácia de manipulação constatou o predomínio de três fitoterápicos mais vendidos que foram: Hypericum perforatum, Ginkgo biloba e Valeriana officinalis (VIEIRA, et al,. 2010). A análise comparativa demonstrou a discordância entre os fitoterápicos mais vendidos entre os dois estabelecimentos.

Tabela 2: Os Cinco Fitoterápicos mais prescritos por período anual na farmácia.

\begin{tabular}{|c|c|c|}
\hline Nome Científico & Nome Popular & Ano \\
\hline Aesculus hippocastanum & Castanha da Índia Ext.Seco & 2014 \\
\hline Vitis vinífera & Óleo Semente de Uva & 2014 \\
\hline Tribullus terrestres & Abre-os-olhos & 2014 \\
\hline Carapa guaianesis Aubl & Óleo de Andiroba & 2014 \\
\hline Equisetum arvense L. & Cavalinha Extrato Seco & 2014 \\
\hline Aesculus hippocastanum & Castanha da Índia Ext. Seco & 2015 \\
\hline Vitis vinífera & Óleo de Semente de Uva & 2015 \\
\hline Equisetum arvense L. & Cavalinha Extrato Seco & 2015 \\
\hline Tribullus terrestres & Abre-os-olhos & 2015 \\
\hline Carapa guaianesis Aubl & Óleo de Andiroba & 2015 \\
\hline Aesculus hippocastanum & Castanha da Índia Ext.Seco & 2016 \\
\hline Tribullus terrestres & Abre-os-olhos & 2016 \\
\hline Vitis vinífera & Òleo Semente de Uva & 2016 \\
\hline Carapa guaianesis Aubl & Óleo de Andiroba & 2016 \\
\hline Equisetum arvense L. & Cavalinha Extrato Seco & 2016 \\
\hline Tribullus terrestres & Abre-os-Olhos & 2017 \\
\hline Aesculus hippocastanum & Castanha da Índia Ext. Seco & 2017 \\
\hline Vitis vinífera & Òleo Semente de Uva & 2017 \\
\hline Calendula officinalis $L$ & Calêndula Ext.Glicol & 2017 \\
\hline Aloe vera $L$. & Babosa Ext.Glicol & 2017 \\
\hline Aloe vera $L$. & Babosa Ext.Glicol & 2018 \\
\hline Calendula officinalis $L$ & Calêndula Ext.Glicol & 2018 \\
\hline Vitis vinífera & Òleo Semente de Uva & 2018 \\
\hline Aesculus hippocastanum & Castanha da Índia Ext.Seco & 2018 \\
\hline Matricaria recutita $L$. & Camomila Ext.Glicol & 2018 \\
\hline
\end{tabular}

Fonte: Pesquisa dos autores, 2018. 


\section{Formas Farmacêuticas Mais Utilizadas nas Preparações}

Dentre os cinco fitoterápicos mais utilizados no período em questão, soma-se um total de 7.973 fórmulas vendidas, sendo que 4.283 pertencentes ao laboratório de manipulação de Sólidos e 3.690 fórmulas pertencentes ao laboratório de Líquido-Semissólidos. Conforme expresso na tabela 3 .

Tabela 3: Distribuição dos medicamentos fitoterápicos por setor.

\begin{tabular}{|l|c|c|}
\hline \multicolumn{1}{|c|}{ Setor } & Frequência & Porcentagem (\%) \\
\hline Laboratório de Sólidos (cápsulas) & 4.283 fórmulas & 53,71 \\
\hline Laboratório de Líquido/Semissólidos & 3.690 Fórmulas & 46,29 \\
\hline Total & 7.973 fórmulas & 100 \\
\hline
\end{tabular}

Fonte: Pesquisa dos autores, 2018.

Observa-se que o setor de laboratório de sólidos apresenta uma maior frequência de consumo de produtos fitoterápicos que em sua maioria são compostos por cápsulas. De acordo com estudos realizados por (VIEIRA, et al, 2010), corrobora com referida análise, pois $72,8 \%$ das fórmulas foram na forma farmacêutica em cápsula. Outro estudo realizado por (DAMASCENO, L, M. 2013) constatou um percentual de 59\% na mesma forma farmacêutica.

\section{Avaliação da Evolução das vendas no período de 2014 a 2018}

Na coleta das informações sobre a evolução do número de vendas, podemos destacar um declínio nos cinco últimos anos, tal fato pode ser associado a vários fatores como, por exemplo, crise financeira no país, concorrência entre farmácias, falta do produto no mercado farmacêutico, adesão aos medicamentos alopáticos, redução da publicidade da empresa etc. No gráfico 2 abaixo podemos identificar a redução em cada ano. Podemos perceber que no ano de 2014 a quantidade de fórmulas foi de 4.202 e no ano de 2018 caiu para 2.698 tendo uma redução de 1.504 fórmulas. Entre os anos de 2015, 2016 e 2017 não houve grandes diferenças significativas. 
Gráfico 2 Avaliação da Evolução das vendas no período 2014 a 2018

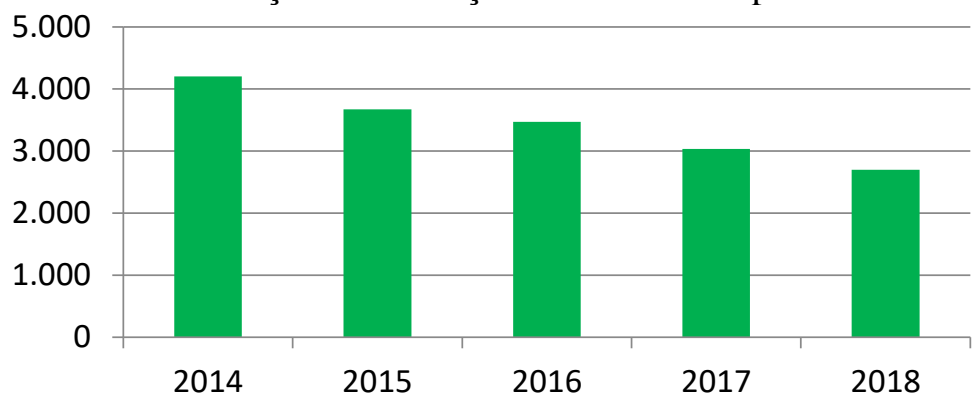

Fonte: Pesquisa dos autores, 2018.

De acordo com análise de registros nas vendas, foi verificada uma redução gradativa dos fitoterápicos. Tal fato pode ser justificado devido à crise financeira que o país alcançou a partir de 2014. Tendo como ponto paralelo a inserção da fitoterapia ao SUS. Tendo como base fundamentada hipóteses de análise de mercado.

Ao longo dos últimos quatro anos, o setor mostrou sua resiliência. Como primeiro impacto da instabilidade na economia no país, houve queda de 4,8\% no faturamento de acordo com o PIB nacional. Atualmente o setor passa por um aquecimento para em breve recuperar o desempenho obtido antes da crise (YASBEK, C. 2018).

\section{Considerações Finais}

De acordo com os resultados expostos ficou claro concluir a evidência do perfil da prescrição de fitoterápicos no período de cinco anos na farmácia magistral em questão, onde podemos integrar os resultados encontrados como um fator positivo para a melhoria da qualidade dos serviços prestados e avaliar uma possível estratégia para elevar seus rendimentos na terapia com os fitoterápicos. Esta estratégia pode ser relacionada a treinamento de toda a equipe como forma de conhecimento específico da literatura de todos os fitoterápicos que contém na empresa. Facilitando o trabalho do farmacêutico como profissional da saúde, entrando em concordância com as tendências das políticas pública, que é desenvolver maior integração entre profissionais da saúde com a população. 


\section{Referências}

ALMEIDA, E. R. As plantas medicinais brasileiras. São Paulo: Hemus, 1993.

BRUNO, I.; NALDI, N. O. O milagre das plantas. São Paulo: Editora Atheneu, 1998.

BRASIL, 2006. Resolução - RDC n⿳10135 de 29 de maio de 2003. Aprova Regulamento Técnico para Medicamentos Genéricos. Diário oficial da República Federativa do Brasil, Brasília, DF.

BRASIL. Ministério da Saúde. Portaria No 971, de 03 de maio de 2006. Aprova a Política Nacional de Práticas Integrativas e Complementares (PNPIC) no Sistema Único de Saúde. Diário Oficial da União, Poder Executivo, Brasília, DF, 04 mai. 2006a. Seção 1, p. 20. Disponível em: http://bvsms.saude.gov.br/bvs publicações/pnpic.pdf . Acesso em: Outubro de 2018.

BRASIL. Agência Nacional de Vigilância Sanitária (ANVISA). Resolução RDC nº 13, de 14 de março de 2013. Dispõe sobre as boas práticas de fabricação de Produtos Tradicionais Fitoterápicos. Diário Oficial da União. Disponível em: http://www.in.gov.br/visualiza/ index.jsp?data=15/03/2013\&jornal=1\&pagina=50\&totalArquivos=200 . Acesso em: Outubro de 2018.

DAMASCENO, L.M. Perfil dos Medicamentos Fitoterápicos Mais Comercializados em Farmácia Magistral do Município de João Pessoa, 2013.

DI STASI, L.C.(org.) Plantas Medicinais: Arte e Ciência. Um guia de estudo interdisciplinar. São Paulo. Ed. UNESP. 230 p., 1996.

LIMA, L. Fitoterápicos e usos de plantas medicinais. Jornal da Unesp, ano XVI, n. 166, 2006. Disponível em: http://www.unesp.br/aci/jornal/166/farmacologia.htm . Acesso em: abril de 2018.

MASTROIANNI, P.C. Análise dos aspectos legais das prescrições de medicamentos. Rev Ciênc Farm Básica Apl. v. 30, n. 2, 173-176, 2009.

RIBEIRO, A.Q.; LEITE, J.P.V.; DANTAS-BARROS, A.M.. Perfil de utilização de fitoterápicos em farmácias comunitárias de Belo Horizonte sob a influência da legislação nacional. Rev. Bras. Farmacogn. v. 15, n. 1, 2005.

SILVIA, BANDEIRA.; OLIVEIRA. Fitoterapia Racional: um guia de fitoterapia para as ciências da saúde. 1. ed. bras. Barueri: Editora Manole, 2012.

VIEIRA, SILVIA C. HEREDIA; SÓLON, SORAYA; VIEIRA, MARIA DO C.; ZÁRATE, NÉSTOR A. HEREDIA. Levantamento de fitoterápicos em farmácias magistrais de Dourados-MS. Rev. Bras. Farmacogn. v. 20 n. 1, 2010.

\section{Como citar este artigo (Formato ABNT):}

MORAES, Marcelo Batista; MARQUES, Matheus Santos. Perfil da Prescrição de Fitoterápicos em uma Farmácia de Manipulação de Vitória da Conquista-BA entre 2014 a 2018. Id on Line Rev.Mult. Psic., 2019, vol.13, n.43, p. 76-86. ISSN: 1981-1179.

Recebido: 13/11/2018;

Aceito: $19 / 11 / 2018$ 